\title{
A Simple Technique for Measuring Particle Size Distributions during Suspension Polymerization
}

\author{
Giovanni Polacco, ${ }^{\dagger}$ Carlo Basile, Maurizio Palla, and Daniele Semino \\ Department of Chemical Engineering, University of Pisa, \\ Via Diotisalvi, 2-56126 Pisa, Italy
}

(Received February 28, 2000)

\begin{abstract}
The techniques actually available for the measurement of particle size distributions are mainly used for liquid-liquid or solid-liquid dispersions in non-reactive systems and there is a lack of experimental data for the dynamics of these distributions under reactive conditions. In the case of suspension polymerization this is mainly related to the optical properties of the reaction medium and to the difficulties inherent in the measurements of properties which rapidly change with time. In this paper a new procedure is described to measure the dynamics of particle size distributions during the course of a suspension polymerization. This technique is based on the ability of an agarose solution, when mixed with a sample of the polymerizing suspension, to transform it into a gel, thereby freezing its morphology. The polymerization can thus be completed under "static" conditions, that is without breakage and coalescence events, and the measurements can be performed in a conventional way for solid particles. The procedure is very simple and can be applied to any process with water as suspending agent. As an example experimental data are shown for the batch suspension polymerization of methylmethacrylate carried out in different operating conditions.
\end{abstract}

KEY WORDS Particle Size Distribution / Suspension Polymerization / Poly(methylmethacrylate) /

Suspension polymerization is an important process in the industrial polymerization practice. ${ }^{1,2}$ This process presents some advantages like: a) the ability of removing the heat evolved during the reaction; b) the possibility of maintaining a good fluidity of the reaction medium during the course of the polymerization; c) the low level of impurities in the final product, d) the low separation costs and e) the production of polymer particles with dimensions that can be appropriate for different applications such as expandable polystyrene, chromatographic separations, ion-exchange resins, acrylic cements for bone surgery, etc. In particular with regard to the latter applications, the effectiveness of the produced suspension strongly depends on the particle size distribution (PSD) for which the available methodologies for on-line measurements and predictive estimation are not well established. The techniques to measure PSD ${ }^{3}$ are: 1 ) sieving, centrifugation and sedimentation (used for solid particles), 2) electrical sensing, spectroscopy, microscopy and light scattering. The experimental data that can be found in the literature, as far as the measurements of PSD in liquid-liquid dispersions are concerned, refer to steady state ${ }^{4-10}$ or transient ${ }^{11-14}$ conditions of nonreacting systems. These data mainly refer to nonreacting liquid-liquid dispersion while, in the case of reacting systems, generally only the final PSD is available. ${ }^{15-18}$ The PSD evolution during suspension polymerization is presented in very few cases and this is probably due to opacity of the reaction mixture which makes inadequate the optical measurement systems.

In this paper a new simple and inexpensive technique of general applicability is presented for discontinuous determination of PSD during suspension polymerization. The idea is the following: a sample is taken, it is put in a solution of agarose in water and cooled in liquid nitrogen in order to obtain a gel. In this way the sus- pended particles are trapped in fixed positions and further changes in PSD are avoided. The sample temperature is increased again without breaking the gel and the polymerization continues in this "static" condition. PSD is analyzed after the end of the polymerization, when the particles are no longer affected by breakage and coalescence phenomena. The technique can be adopted for any polymerization carried out using water as suspending phase. In the present study the polymerization of methyl methacrylate (MMA) was chosen.

From a phenomenological point of view, PSD is the result of a dynamic balance between breakage and coalescence events. Both phenomena are affected by a number of operating conditions and PSD strongly changes during the polymerization because of changes in viscosity and interfacial tension of the dispersed phase. In particular, a sharp variation of these properties occurs during gel and glass effect. Gel and glass effects are typical phenomena that appear during the course of bulk and suspension polymerization of many monomers. The gel (or Trommsdorf) effect ${ }^{19-21}$ is mainly related to the increase in viscosity of the reacting phase. In the case of MMA polymerization, the mobility of the growing chains starts to decrease for a monomer conversion of about 30 $-40 \%$ and the termination event becomes diffusion controlled. On the other side, at this stage the viscosity still does not affect significantly the propagation event because the small monomer molecules maintain a good mobility. Therefore, reaction rate, viscosity, molecular weight and heat generation increase drastically in the range of monomer conversion $40-80 \%$. As a consequence, the gel effect does not affect only the kinetic of the reaction but also the breakage and coalescence phenomena and therefore the PSD of the suspended phase. In particular, the high viscosity favors coalescence with respect to droplet breakage and the mean diameter of

${ }^{\dagger}$ To whom correspondence should be addressed (E-mail: g.polacco@ing.unipi.it). 
the particles tends to increase. At high conversions also the propagation step becomes diffusion controlled (glass effect) and the reaction rate tends rapidly to zero. At this point the suspended phase is in an almost solid state and both breakage and coalescence stop so that the PSD remain unchanged till the end of reaction. A large number of models have been proposed in the last decades to describe gel and glass effects. Reviews regarding these models are available in the literature.$^{22-25}$

In what follows, the new technique for PSD measurement is accurately described and a number of experimental distributions obtained for the suspension polymerization of MMA carried out under different operating conditions are presented.

\section{EXPERIMENTAL}

\section{Materials}

MMA, benzoyl peroxide (BPO, 97\% purity used as radical initiator), 2-hydroxyethyl cellulose (HEC, used as suspending agent) and agarose were provided by Aldrich (Aldrich Chemie GmbH, Grünwalder WEG 30 D-32041, Deisenhofen, Germany).

The monomer was purified through several washings with $10 \% \mathrm{NaOH}$ (with this concentration no hydrolysis phenomena occur during this stage of the purification) and distilled water, drying with $\mathrm{CaCl}_{2}$ and successive vacuum distillation. BPO was purified twice by precipitation with methanol out of a chloroform solution. 2hydroxyethyl cellulose and agarose were used as received.

\section{Polymerizations}

Suspension polymerizations of MMA were carried out in a glass reactor equipped with a thermostatting jacket and four baffles; the volume of the reactor was $1 \mathrm{~L}$. The stirrer was a six-blade $45^{\circ}$ modified Rusthon type impeller with an Eurostar (IKA Labortechnik D-79219, Staufen, Germany) digital engine, which guarantees a constant stirring speed even when the viscosity of the reaction medium varies; the blade diameter was $5.5 \mathrm{~cm}$. All polymerizations were carried out as follow:

- The suspending solution of HEC in water was feed to the reactor and the latter was fluxed with nitrogen for 90 min under stirring.

- The monomer was heated separately to the same temperature of the suspending solution, mixed with BPO and fed to the reactor.

- The temperature in the reactor was maintained within $\pm 1^{\circ} \mathrm{C}$ of the set point. More significant deviations from the desired temperature were observed only after completion of the reaction, when the heat generation vanishes in a very short time.

- During the course of the polymerization, samples of 2 and $4 \mathrm{~mL}$ (for PSD and gravimetric analysis, respectively) were removed close to the impeller blades.

\section{Procedure for PSD Measurement}

The idea is derived from the "gelled polymerization" 26,27 which is a "static" (carried out without stirring) suspension polymerization where the suspending phase is a gel of water and agarose; the developed procedure is similar to the one previously tested for an agitated suspension polymerization of MMA carried out using agarose as suspending agent. ${ }^{28,29}$

One of the main characteristics of agarose is its mechanical-thermal hysteresys. Agarose can be dissolved in hot water and a clear solution is obtained; if the temperature of the solution is reduced, a consistent gel is formed at about $40^{\circ} \mathrm{C}$. Once formed, the gel remains stable up to a temperature of about $90^{\circ} \mathrm{C}$. Therefore, if a suspension of a monomer is trapped in the gel, the thermal stability of the latter prevents the mobility of both phases so that any contact between the particles during the polymerization stage is avoided.

On the basis of these phenomena, a new sampling procedure was developed in order to investigate the dynamics of PSD of the suspension under reacting conditions even when conventional suspending agents were used. An initial sample and all the other samples which were removed during the course of polymerization were pretreated and analyzed as follows:

1. The liquid sample (about $2 \mathrm{~mL}$ ) was stored in a test tube containing $4 \mathrm{~mL}$ of a $2 \mathrm{wt} \%$ agarose solution in distilled water (this solution was maintained at $50^{\circ} \mathrm{C}$ in order to stay in a liquid state). The test tube was then gently stirred for a few seconds, in order to mix the sample and the agarose solution, and then it was immediately put in liquid nitrogen for about $5 \mathrm{~s}$. In this way water solidification was avoided while a gel was formed so as to trap the suspended particles in fixed positions. This step required no more than 20 $30 \mathrm{~s}$ during which HEC and agarose guarantee the stability of the monomer suspension.

2. After the gel was consolidated the test tube was transferred in a thermostatted bath at $60^{\circ} \mathrm{C}$ and kept there overnight; the initiator started to decompose again and the polymerization was therefore completed without breaking the gel, so that no coalescence or breakage occurred. The final suspended phase was hence composed of microspheres of poly(methyl methacrylate) with a diameter distribution identical to that of the removed samples with the exception of the volumetric shrinkage due to reaction. Although the monomer is slightly soluble in water, no significant degradative diffusion process ${ }^{30}$ is expected to take place in the agarose gel.

3. The gel was heated at $100^{\circ} \mathrm{C}$, broken mechanically if necessary, taken out of the test tube and put into a beaker containing $400 \mathrm{~mL}$ of boiling water; after 30 min the gel was completely dissolved. A slow cooling (overnight) allowed the decantation of the microspheres on the bottom of the beaker while the agarose formed a colloidal suspension that could be easily removed.

4. Water was removed from the beaker and replaced with fresh water; then step 3 was repeated twice or three times until agarose was completely removed.

5. The volumetric diameter distribution of the microspheres (fraction of the total volume belonging to each class of diameters) was finally measured 
Table I. Base-case operating conditions

\begin{tabular}{lc} 
Reaction temperature $\left({ }^{\circ} \mathrm{C}\right)$ & 70 \\
\hline Water to monomer weight ratio & $5 / 1$ \\
\hline Initiator concentration $(\mathrm{mol} / \mathrm{L}$ of monomer $)$ & 0.0178 \\
\hline HEC $(\mathrm{g} / 100 \mathrm{~g}$ of water $)$ & 0.6 \\
\hline Stirring speed(rpm) & 750 \\
\hline
\end{tabular}

Table II. Operating conditions of the different tests

\begin{tabular}{cccc}
\hline Test & $\mathrm{rpm}$ & $\mathrm{H}_{2} \mathrm{O} / \mathrm{MMA}(\mathrm{w} / \mathrm{w})$ & $\%$ HEC \\
\hline 2 & 500 & $5 / 1$ & 0.6 \\
\hline 3 & 1000 & $5 / 1$ & 0.6 \\
\hline 4 & 750 & $6 / 1$ & 0.6 \\
\hline 5 & 750 & $4 / 1$ & 0.6 \\
\hline 6 & 750 & $5 / 1$ & 0.8 \\
\hline 7 & 750 & $5 / 1$ & 0.4 \\
\hline
\end{tabular}

through a MALVERN 2605 lc: a sample of about 200 mg of microspheres was put in a solution of $\mathrm{Na}_{3} \mathrm{PO}_{4}$ (in order to avoid particle agglomeration) in water and analyzed while keeping the solution under stirring.

\section{Gravimetric Analysis}

Monomer conversion was calculated by gravimetric analysis of samples taken from the reactor at specific time intervals. Firstly the samples $(4 \mathrm{~mL})$ were poured into test tubes containing methanol and an inhibitor (hydroquinone) (methanol/sample volume ratio $=10$ ), then the tubes were centrifuged and the solid washed with methanol and centrifuged again. Finally the solid was dried till constant weight.

\section{Scanning Electron Microscopy}

Microspheres obtained by the PSD sampling procedure were analyzed by a scanning electron microscope Jeol T300. Samples were dried, sputtered with gold and observed at $10 \mathrm{kV}$.

\section{RESULTS AND DISCUSSION}

Seven different tests were performed. The operating conditions of the first one, referred in the following as the base-case, are reported in Table I. The other six tests were undertaken by maintaining the same jacket temperature and initiator concentration of the base-case and varying one among the following parameters at a time: stirring speed, water to monomer weight ratio and HEC concentration, as reported in Table II.

\section{Base-Case}

Samples were taken at time $t=45,60,70,80,90,95$, 100,120 , and $180 \mathrm{~min}$. The frequency of sampling was increased in the time interval when the gel effect was taking place. As discussed above, it is in this period that the polymerizing system undergoes the most significant changes. Conversion vs. time is shown in Figure 1. It can be seen that the gel effect starts after about $80 \mathrm{~min}$ while the glass effect starts after $100 \mathrm{~min}$. In the figures the results are reported till $t=120 \mathrm{~min}$ because after

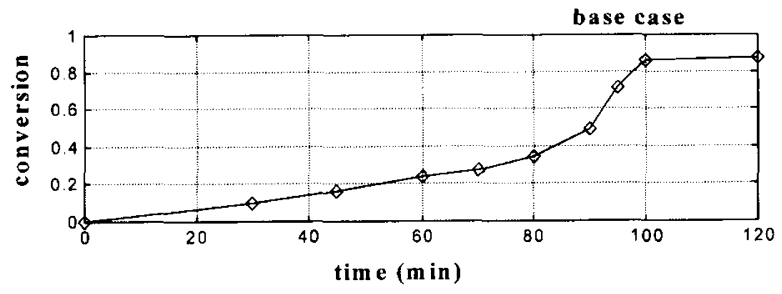

Figure 1. Conversion versus time curve for base-case conditions.

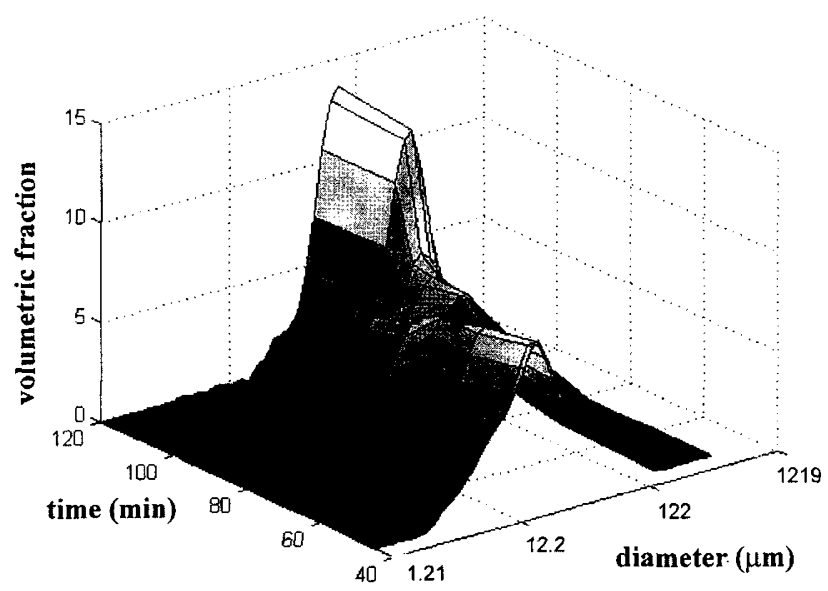

Figure 2. Particle size distributions for base-case conditions.
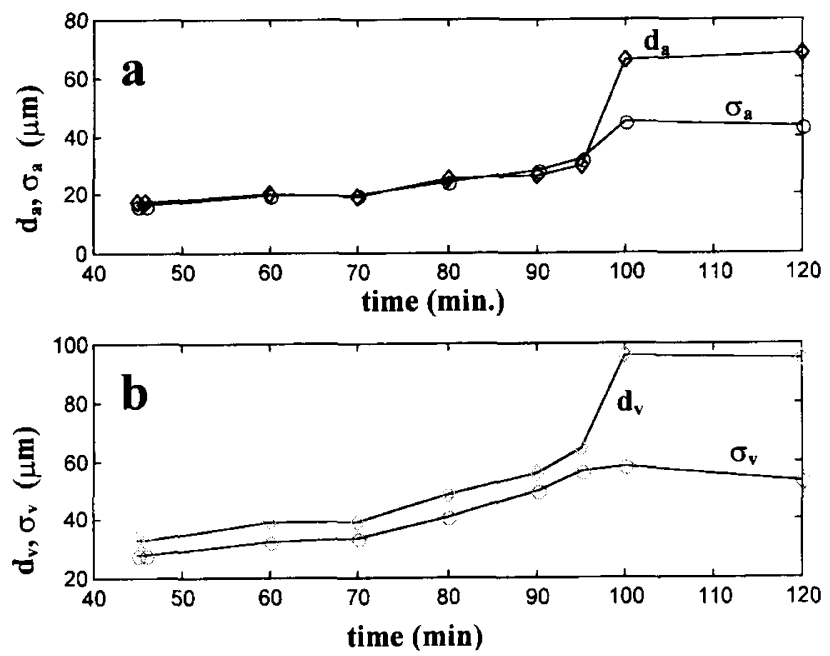

Figure 3. Sauter diameter (a), mean volume diameter (b) and their variances as a function of time for base-case conditions.

this time the reaction was almost over and no more variations in PSD are expected. The obtained particle size distributions are plotted in Figure 2 as a function of time. Figure 3 shows the mean Sauter diameter $\left(d_{\mathrm{a}}\right)$, the mean volumetric diameter $\left(d_{\mathrm{v}}\right)$ and their variances $\left(\sigma_{\mathrm{a}}\right.$, $\sigma_{\mathrm{v}}$ ) defined as follows:

$$
\begin{aligned}
& d_{\mathrm{a}}=\frac{\sum_{i} n_{i} d_{i}{ }^{3}}{\sum_{i} n_{i} d_{i}{ }^{2}}, \sigma_{\mathrm{a}}{ }^{2}=\frac{\sum_{i} n_{i} d_{i}{ }^{2}\left(d_{i}-d_{\mathrm{a}}\right)^{2}}{\sum_{i} n_{i} d_{i}{ }^{2}} \\
& d_{\mathrm{v}}=\frac{\sum_{i} n_{i} d_{i}{ }^{4}}{\sum_{i} n_{i} d_{i}{ }^{3}}, \sigma_{\mathrm{v}}{ }^{2}=\frac{\sum_{i} n_{i} d_{i}{ }^{3}\left(d_{i}-d_{\mathrm{a}}\right)^{2}}{\sum_{i} n_{i} d_{i}{ }^{3}}
\end{aligned}
$$


(a)

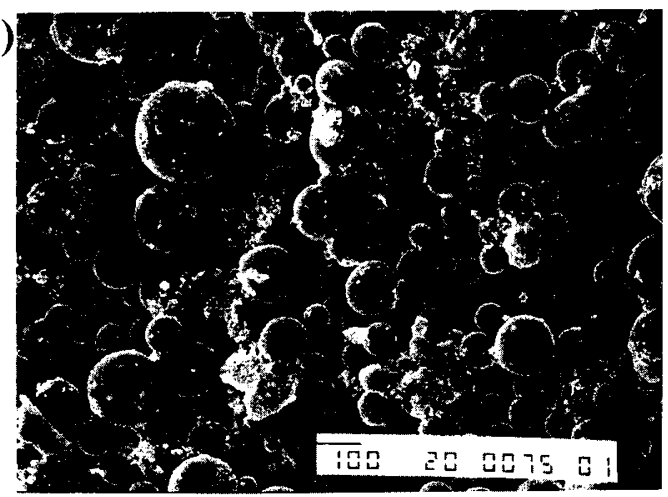

(c)

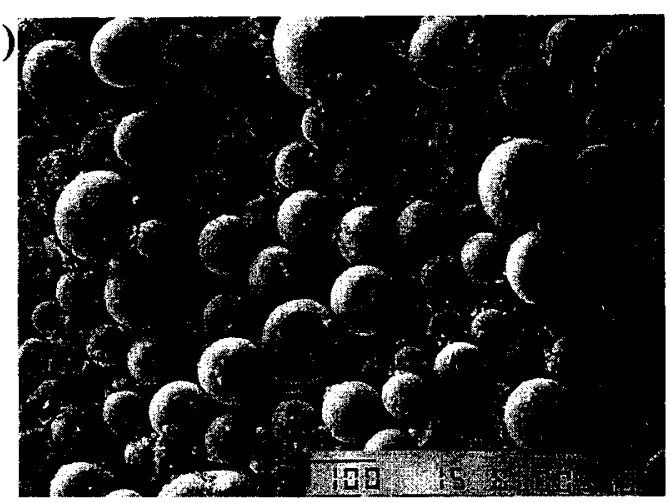

(e)

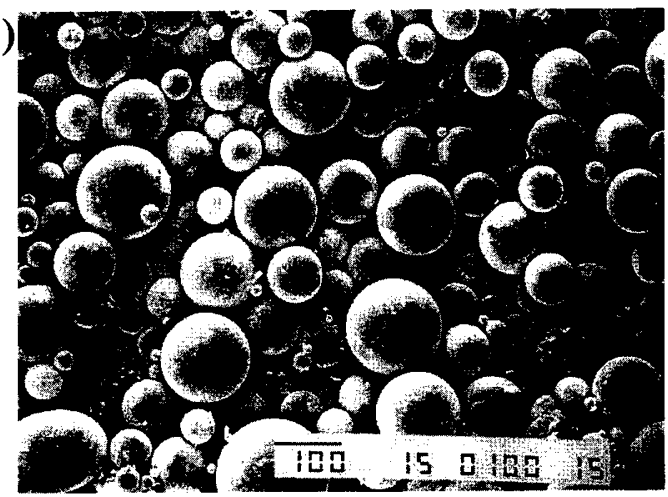

(g)

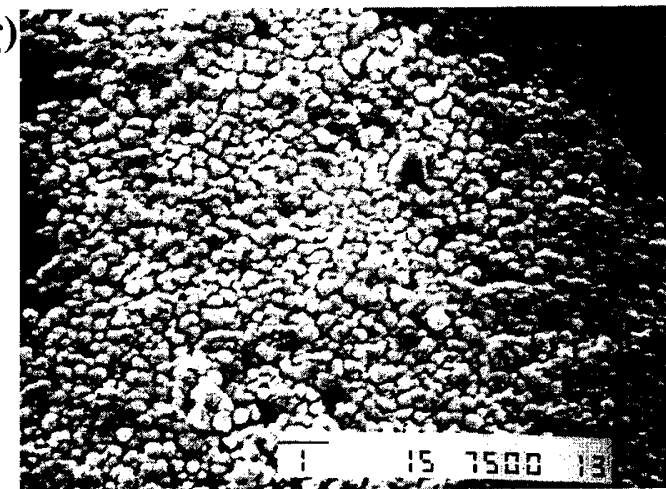

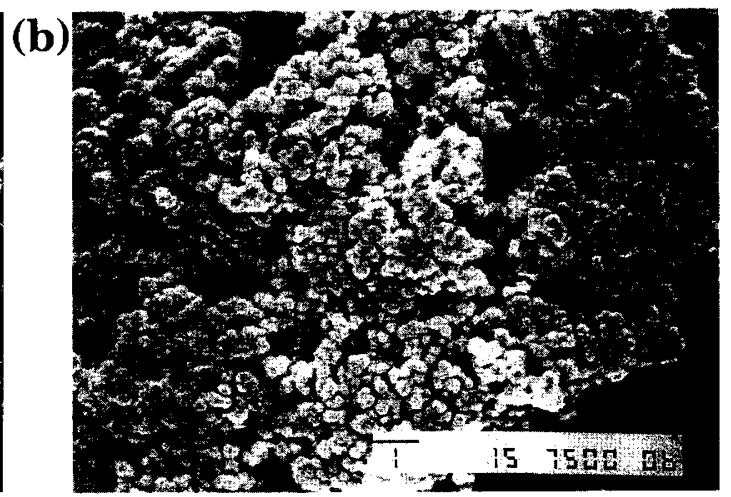

(d)

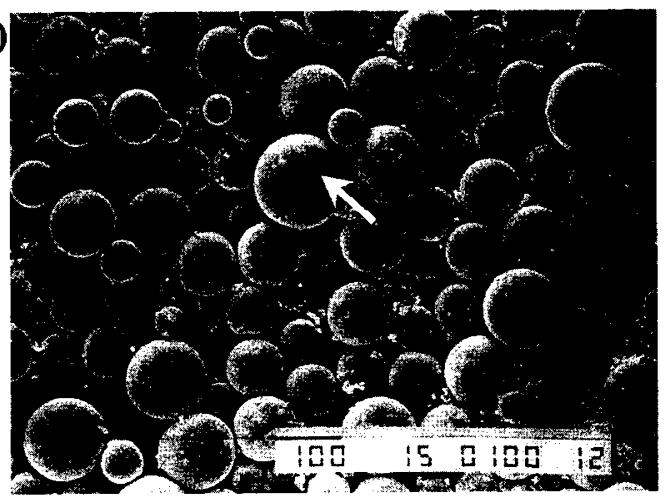

(f)

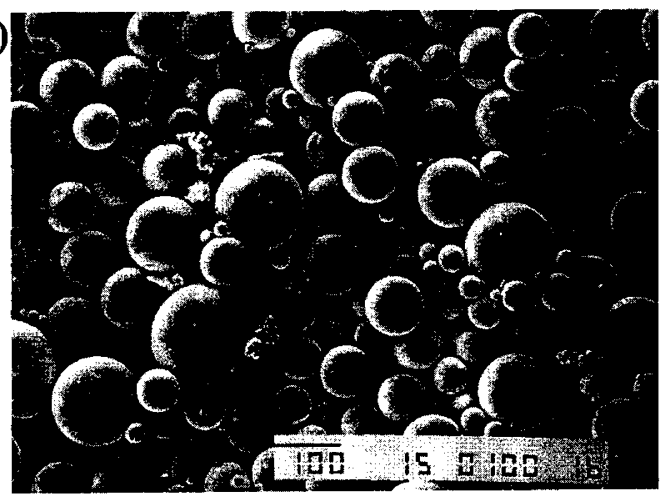

Figure 4. SEM images of the samples taken during the base-case polymerization: a) $t=60 \mathrm{~min}$, bar $=$ $100 \mu \mathrm{m}$; b) magnification of a detail of Figure $4 \mathrm{a}$, bar $=1 \mu \mathrm{m}$; c) $t=80 \mathrm{~min}, \mathrm{bar}=100 \mu \mathrm{m}$; d) $t=90 \mathrm{~min}$, bar $=100 \mu \mathrm{m}$; e) $t=100 \mathrm{~min}, \mathrm{bar}=100 \mu \mathrm{m} ;$ f ) $t=$ $120 \mathrm{~min}, \mathrm{bar}=100 \mu \mathrm{m} ; \mathrm{g})$ magnification of the surface of the particle indicated with an arrow in Figure $4 \mathrm{~d}, \mathrm{bar}=1 \mu \mathrm{m}$.
It can be seen that the first distribution is bimodal, with the two peaks centered at $d=23.7 \mu \mathrm{m}$ and $d=65.7$ $\mu \mathrm{m}$. During the first stage of polymerization, the distribution changes and the first peak decreases while the second one increases gradually so that the distribution approach an unimodal shape. This indicates that in the initial stage of the reaction, coalescence prevails on breakage especially for small particles. This is confirmed by the slow increase of the mean diameter. When the re- action enters the gel-effect region, the same phenomena becomes more relevant and a strong variation in PSD takes place in a few minutes so that the distribution result unimodal with a high peak centered at $d=90 \mu \mathrm{m}$. In about five minutes $(t=95-100 \mathrm{~min}$ ) the Sauter diameter shifts from 30 towards $66 \mu \mathrm{m}$ while the relative variance still increases but much less steeply.

SEM analysis confirms these results. Figure $4 \mathrm{a}$ shows an image of the sample taken at time $t=60 \mathrm{~min}$. It can 
be observed that particles diameter ranges from about 10 to $150 \mu$. In addition, the photograph shows the presence of irregular particles which appear like a powder; however a strong magnification of these particles (Figure $4 \mathrm{~b}$ ) demonstrates that they are composed of a very big number of aggregate "nanospheres" with a diameter less than $1 \mu$. These small particles are likely to be related to an emulsion polymerization taking place in parallel to the suspension polymerization. Since MMA is an hydrophilic monomer, this unfavorable effect can not be avoided unless some water inhibitors is added to the aqueous phase. Unfortunately, these "nanoparticles" can give some problems for the experimental determination of PSD: if the particles are aggregate an experimental error would occur during the measurements performed with the Malvern particle sizer; on the contrary, if they are separated they result undetectable because of their small size. In any case, the obtained distribution can be considered precise enough because of the small amount of aggregates. Moreover, as shown by the PSD curves, the number of small particles gradually decreases during the course of polymerization as confirmed by the images of the other samples. Figures $4 \mathrm{c}-4 \mathrm{f}$ show the SEM images of samples taken at times $80,90,100,120 \mathrm{~min}$, respectively. It is clear from these pictures that the particles become greater and "cleaner" with time and almost no aggregates are detectable after the onset of the gel effect. Figure $4 \mathrm{~g}$ shows a magnification of the surface of the particle indicated with an arrow in Figure $4 \mathrm{~d}$. The picture shows a lot of nanoparticles bonded on this surface suggesting an intermediate phase of coalescence. Anyway the last samples are composed of spherical, clean particles with dimensions in the expected range.

\section{Non Base-Cases}

As stated above, a number of further tests were undertaken in order to examine the role played by the main operating conditions which directly affect the breakage and coalescence phenomena without changing the kinetic of the polymerization reaction. Therefore, the timeconversion curves of cases 2-7 do not differ from the one reported in Figure 1. With regard to the particle dimensions, from a qualitative point of view the behavior is similar in all cases, with small PSD variations during the first stage of reaction, big changes during the gel effect and a progressive increase of the mean Sauter and volumetric diameters. On the contrary, from a quantitative point of view the distributions show different values of the peaks, of their position and of the corresponding mean values. The measured volumetric diameter distributions obtained for cases 2 (stirring speed $=500 \mathrm{rpm}$ ), and 3 (stirring speed $=1000 \mathrm{rpm}$ ) are reported in Figure 5 , and 6 respectively. In order to compare the changes in PSD due to the different operating conditions, Figure 7 shows the mean Sauter diameters in a more compact way. In particular, it can be seen that the mean Sauter diameter for case 2 result greater than that for base-case and case 3 for all times, while the base-case and case 3 differ significantly only after the onset of the gel-effect.

For the sake of brevity, with regard to the other polymerizations only the PSD obtained for cases 5 and 6 (Figures 8 and 9, respectively) are reported. As stated above, the PSD is still qualitatively similar to that of

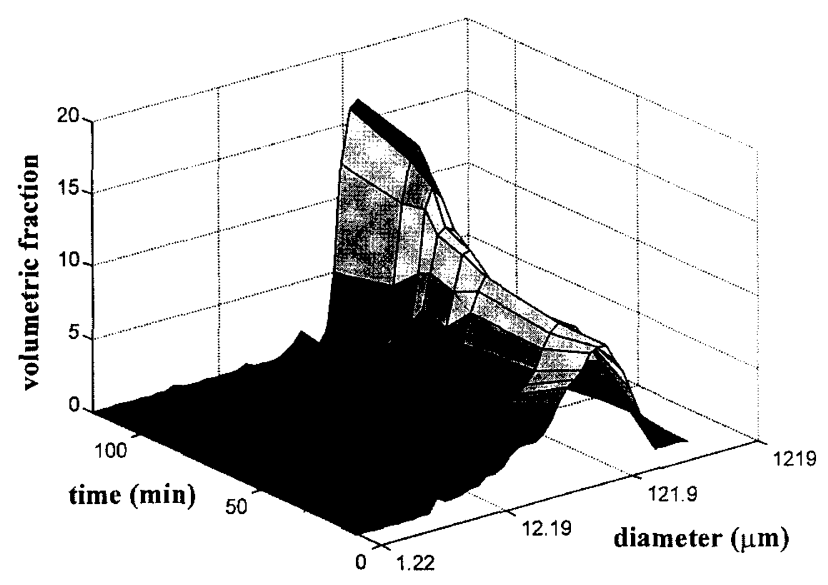

Figure 5. Particle size distributions for case 2.

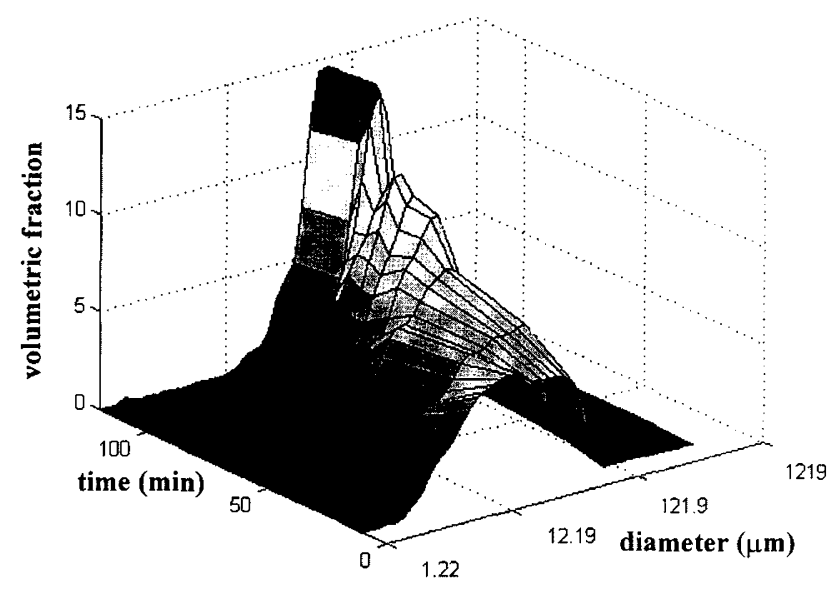

Figure 6. Particle size distributions for case 3.

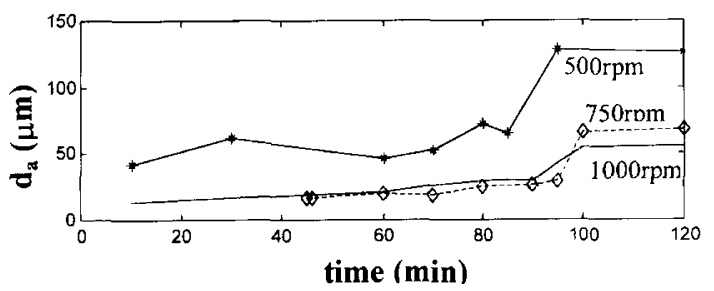

Figure 7. Sauter diameter as a function of time for base-case, case 2 and 3.

base-case. This confirms the observed trend of significant predominance of the coalescence phenomena when the viscosity increases due to the presence of a higher polymer percentage in the particles.

\section{CONCLUSIONS}

A new technique has been described for measuring particle size distributions during the course of a suspension polymerization. The technique has been derived from a previously developed one, ${ }^{28,29}$ whose applicability was limited to polymerizations carried out using water as suspending phase and agarose as suspending agent. It has been shown how agarose, which is needed in order to obtain a very stable gel in a water solution over a wide range of temperature, can be used as an "external" com- 


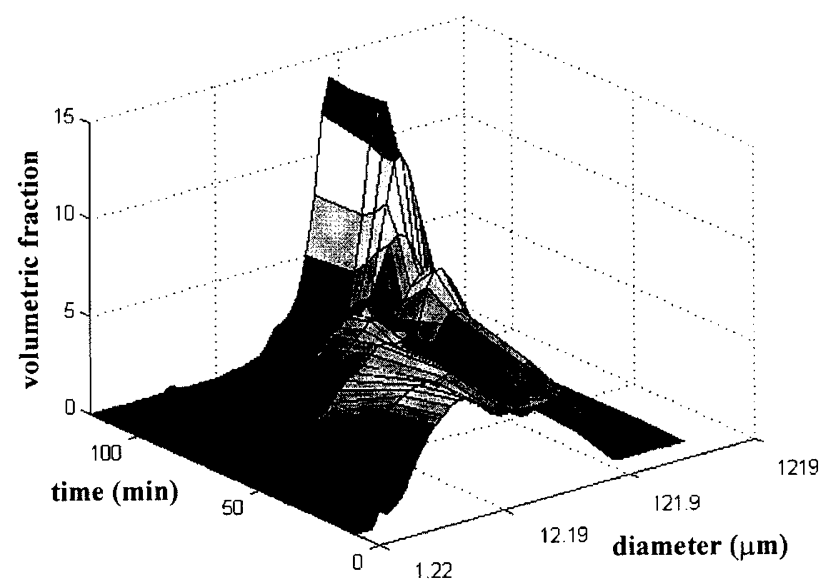

Figure 8. Particle size distributions for case 5.

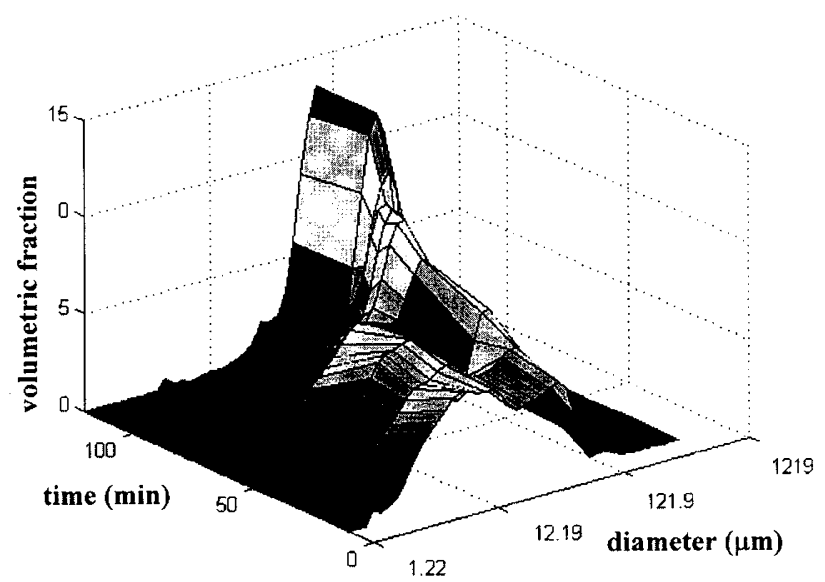

Figure 9. Particle size distributions for case 6.

ponent so that the technique can be applied also to polymerization carried out with any desired suspending agent. Therefore, the technique can be applied to any kind of suspension process in which water is used as suspending phase and monomer and initiator are able to operate at any temperature below $90^{\circ} \mathrm{C}$ (that is the highest temperature which guarantees the gel stability). Of course, this technique can be further extended to other types of polymerization (e.g., with different temperature range or different suspending phase) simply using an appropriate system able to form in a few seconds a gel with similar stability. The proposed procedure has also the advantage of being simple and inexpensive so that it can be applied extensively without an excessive burden.

Several tests have been performed for batch isothermal suspension polymerizations of methylmethacrylate using water as suspending phase and 2-hydroxyethyl cellulose as suspending agent. Particle size distributions, mean diameters and their respective variances have been measured varying once a time some operating conditions which directly affect the breakage and coalescence phenomena.

Acknowledgments. The authors gratefully acknowledge Mr. Piero Narducci for performing the SEM analysis.

\section{REFERENCES}

1. A. E. Hamielec and H. Tobita, "Ullmann's Encyclopedia of Industrial Chemistry", VCH Publishers, Inc., New York, N. Y., 1992, Vol. A 21 pp 305-428.

2. H. G. Yuan, G. Kalfas, and W. H. Ray, J. Macromol. Sci., Rev. Macromol. Chem. Phys., C31, 215 (1991).

3. E. Vivaldo-Lima, P. E. Wood, A. E. Hamielec, and A. Penlidis, Ind. Eng. Chem. Res., 36, 939 (1997).

4. J. P. Ward and J. G. Knudsen, AIChE J., 13, 356 (1967).

5. S. B. Collins and J. G. Knudsen, AIChE J., 16, 1072 (1970).

6. D. E. Brow and K. Pitt, Chem. Eng. Sci, 25, 577 (1972).

7. C. A. Coulaloglou and L. L. Tavlarides, AIChE J., 22, 289 (1976).

8. C. A. Coulaloglou and L. L. Tavlarides, Chem. Eng. Sci., 32, 1289 (1977).

9. T. Tobin, R. Muralidhar, H. Wright, and D. Ramkrishna, Chem. Eng. Sci., 45, 3491 (1990).

10. E. G. Chatzi and C. Kiparissides, AIChE J., 41, 1640 (1995).

11. A. W. Pacek, and W. Nienow, Trans. Inst. Chem. Eng., 73 Part A, 512 (1995).

12. A. W. Pacek, I. P. T. Moore, A. W. Nienow, and R. V. Calabrese, AIChE J., 40, 1940 (1994).

13. G. Narsimhan, D. Ramkrishna, and J. P. Gupta, AIChE J., 26, 991 (1980).

14. M. Laso, L. Steiner, and S. Hartland, Chem. Eng. Sci., 42, 2437 (1987).

15. M. Konno, K. Arai, and S. Saito, J. Chem. Eng. Jpn., 15, 131 (1982).

16. F. M. Willmouth, D. G. Rance, and K. M. Henman, Polymer, 25, 1185 (1984).

17. B. Törnell and J. Uustalu, J. Appl. Polym. Sci, 35, 63 (1988).

18. Y. Hatate, A. Ikari, K. Kondo, and F. Nakashio, Chem. Eng. Commun., 34, 325 (1984).

19. E. H. Trommsdorf, H. Koenle, and P. Lagally, Makromol. Chem., 1, 169 (1948).

20. R. G. W. Norris and E. F. Brookmann, Proc. Roy. Soc. (London), A171, 147 (1939).

21. G. W. Schultz and G. Harbort, Makromol. Chem., 1, 106 (1947).

22. A. D. Schmidt and W. H. Ray, Chem. Eng. Sci., 36, 1401 (1981).

23. T. J. Tulig and M. V. Tirrel, Macromolecules, 14, 1501 (1981).

24. K. F. O'Driscoll, Pure Appl. Chem, 53, 617 (1981).

25. A. E. Hamielec, Chem. Eng. Commun., 24, 1 (1983).

26. P. Giusti, L. Lazzeri, G. Pizzirani, G. Polacco, C. Rizzo, and M. Palla, J. Mat. Sci.: Mat. In Med., 5, 858 (1994).

27. G. Polacco, D. Semino, and M. Palla, Polym. Eng. Sci., 36 (16), 2088 (1996).

28. G. Polacco, M. Palla, and D. Semino, Polym. Int., 48, 392 (1999).

29. G. Polacco, M. Palla, and D. Semino, Proceedings of DYCOPS 5 IFAC Symposium on Dynamic and Control of Process Systems, Corfù, Greece, June 8-10, 1998, pp 274-279.

30. O. Shinzo, T. Tetsuya, N. Masatoshi, and M.Guang-Hui, J. Appl. Polym. Sci., 63, 931 (1997). 